\title{
Repurposing FDA-Approved Drugs for COVID-19 Using a Data-Driven Approach
}

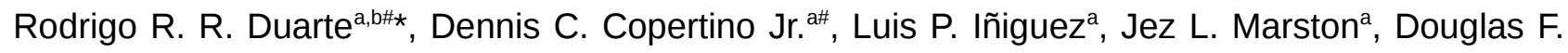
Nixon ${ }^{\mathrm{a}}$, Timothy R. Powell ${ }^{\mathrm{a}, \mathrm{b}}$

a Division of Infectious Diseases, Weill Cornell Medicine, Cornell University, New York, NY, USA.

b Social, Genetic \& Developmental Psychiatry Centre, Institute of Psychiatry, Psychology \& Neuroscience, King's College London, London, UK.

* Correspondence: Dr. Rodrigo R. R. Duarte, Division of Infectious Diseases, Weill Cornell Medicine, Cornell University, Belfer Research Building, Room 527, 413 E. $69^{\text {th }}$ St., New York, NY, 10021, USA. E-mail: rrd4001@med.cornell.edu, Tel: +1 2023807404.

\# Co-first authors.

\section{Summary}

There have been more than 116,000 recorded deaths worldwide to-date caused by the severe acute respiratory syndrome coronavirus type 2 (SARS-CoV-2), the etiological agent of the Coronavirus Disease 2019 (COVID-19), and over 1.8 million individuals are currently infected. Although there are now hundreds of clinical trials for COVID-19, there are currently no effective licensed treatments, while the numbers of infected individuals continue to rise at an exponential rate in many parts of the world. Here, we used a data-driven approach utilizing connectivity mapping and the transcriptional signature of lung carcinoma cells infected with SARS-CoV-2, to search for drugs across the spectrum of medicine that have repurposing potential for treating COVID-19. We also performed chemoinformatic analyses to test whether the identified compounds were predicted to physically interact with the SARS-CoV-2 RNA-dependent RNA polymerase or main protease enzymes. Our study identified commonly prescribed FDA-approved molecules as important candidates for drug repositioning against COVID-19, including flupentixol, reserpine, fluoxetine, trifluoperazine, sunitinib, atorvastatin, raloxifene, butoconazole, and metformin. These drugs should not be taken for treating or preventing COVID-19 without a doctor's advice, as further research and clinical trials are now needed to elucidate their efficacy for this purpose. 


\section{Introduction}

The Coronavirus Disease 2019 (COVID-19), caused by the severe acute respiratory syndrome coronavirus type 2 (SARS-CoV-2), instigated the current global public health crisis that has put many societies on hold. While most individuals recover successfully, COVID-19 is associated with an alarming mortality rate, ranging between 1.5-15.2\% across nations (Baud et al., 2020), which varies according to regional healthcare resources availability ( $\mathrm{Ji}$ et al., 2020), prevalence of comorbidities (Guan et al., 2020a), population age (Onder et al., 2020) and sex (Guan et al., 2020b). There are currently no effective licensed drugs or vaccines to prevent or treat COVID-19 (COVID-19 Clinical Research Coalition, 2020), and the current strategy to prevent the disease includes preventing novel infections through social distancing and community containment, which have been implemented by local and national authorities worldwide (Wu and McGoogan, 2020). However, these measures have profoundly detrimental effects to the economy, and do not represent a feasible long-term solution for the COVID-19 pandemic.

Repurposing commonly prescribed drugs for preventing infection with SARS-CoV-2 or the respiratory symptoms associated with COVID-19 has the potential to fast-track the development of a viable treatment option and to rapidly improve the current global crisis. There are over 360 ongoing clinical trials testing the effectiveness of a variety of compounds against COVID-19 (COVID-19 Clinical Research Coalition, 2020). The majority of drugs being trialed have antiviral activity, and it is logical that these compounds are tested first. However, preliminary results have been variable, and a molecular and data-driven approach should be explored as a way of narrowing down the list of potentially effective medications, from across the spectrum of medicine. One of these approaches is known as 'connectivity mapping', an in silico method that has been used to identify compounds with repurposing potential in diverse areas of medicine (Keenan et al., 2019), including the use of ursolic acid (found in apples) for muscular atrophy (Kunkel et al., 2011), chlorpromazine (antipsychotic) for the treatment of hepatocellular carcinoma (Lee et al., 2015), and celastrol (leptin sensitizer) for the treatment of obesity (Liu et al., 2015), among other examples (Subramanian et al., 2017).

In this study, we used the transcriptional signature associated with SARS-CoV-2 infection in a cell model of COVID-19 comprising of infected carcinoma human alveolar (lung) basal epithelial cells A549 (Blanco-Melo et al., 2020), to identify drugs with repurposing potential for the treatment or prevention of COVID-19. According to Blanco-Melo and colleagues, even though these cells do not express the putative receptor (ACE2) and protease (TMPRSS2) required by SARS-CoV-2, they are able to support viral replication. To identify chemicals with potential for repositioning against COVID-19, we matched the mRNA signature consisting of positively and negatively regulated genes associated with SARS-CoV-2 infection in this cell model to the L1000 repository of cellular transcriptional signatures, using the cloud-based Connectivity Map (CMap) portal (https://clue.io) 
(Subramanian et al., 2017). This repository contains 1.3 million transcriptional profiles associated with the effect of 27,927 perturbagens, including drugs, gene knockdowns and knock-ins, tested in up to 77 cell lines, including the cancer-derived lung cells A549. We also performed chemoinformatic analysis to identify drugs predicted to bind to SARS-CoV-2 key enzymes, and cross-referenced our findings with those from other drug repositioning studies in the literature, to identify FDA-approved drugs with substantial repurposing potential for the treatment of COVID-19.

\section{Methods}

\section{Connectivity mapping}

We used CMap v1.1.1.43, dataset v1.1.1.2, accessed via https://clue.io (Subramanian et al., 2017), to identify drugs with repurposing potential for COVID-19. Genes up- and downregulated in A549 cells after SARS-CoV-2 infection (Blanco-Melo et al., 2020) were input into CMap to search for compounds (in the L1000 database) that elicited reversed transcriptional signatures associated with infection, indicating their potential to reverse viral-related effects on the cells. The connectivity score from CMap is calculated based on the observed enrichment scores in the queried gene lists relative to transcriptional signatures in the L1000 reference database. The score incorporates a nominal $p$-value calculated based on the comparison between the query and reference signatures relative to a null distribution of random queries, using the Kolmogorov-Smirnov enrichment statistic, which is then corrected for multiple testing using the false discovery rate method. These values are converted to tau values $(\tau)$ by comparisons with reference signature queries in the L1000 repository (Subramanian et al., 2017; Subramanian et al., 2005). The authors suggest that drugs with $\tau<-90.00$ are those more likely to reverse the query signature, and conversely, those with $\tau>$ 90.00 are more likely to mimic the query signature. We sought drugs which could reverse the mRNA effects associated with SARS-CoV-2 infection in A549 cells (i.e. those with $\tau<-90.00$ ).

\section{Molecular docking simulations}

We performed molecular docking simulations on FDA-approved small compounds suggested to reverse the transcriptional signature associated with SARS-CoV-2 infection in A549 cells, according to the results of the connectivity mapping analysis. This was performed to identify whether compounds associated with protective effects at the transcriptional level were additionally capable of binding to SARS-CoV-2 enzymes. We analyzed small compounds from the connectivity mapping analysis with $\tau<-90.00$ that were FDA-approved (Corsello et al., 2017) (see https://clue.io/data/REP). We performed molecular docking simulations on the RNA-dependent RNA polymerase (RdRp) (Protein Data Bank [PDB] ID: 6M71), and the main protease (Mpro) (PDB ID: 6Y2E) of SARS-CoV-2, using default settings in the Protein-Ligand ANT System (PLANTS), accessed via https://chemoinfo.ipmc.cnrs.fr/ (Korb et al., 2009). The ligand docking sites were specified as the catalytic sites determined by Zhang et al. (2020) (GIn189) and Gao et al. (2020) 
(Asp623), using an estimated radius of $10 \AA$ around the specified residues. The resulting proteinligand scores (PLANTS scores), calculated using the CHEMPLP algorithm, reflect the energy change when ligands and proteins come together, with values more negative than -80.00 suggesting ligand-protein interactions. The source of the drug structures analyzed is listed in Supplementary Table 1. Protein-ligand visualizations were generated using PyMol 2.3 (https://pymol.org).

\section{Cross-referencing with drugs found in other chemoinformatic studies}

We cross-referenced the compounds from the connectivity mapping analysis $(\tau<-90.00)$ with those suggested for repurposing against COVID-19 according to relevant protein-drug interactions described in the literature, or identified using chemoinformatic analyses (Supplementary Table 2). The drugs suggested by these studies included those identified based on SARS-CoV-2-human protein-protein interactions (Gordon et al., 2020), HIV drugs that bind to the SARS-CoV-1 polymerase or protease enzymes (Chang et al., 2020), or the top FDA-approved drugs that have the potential to bind to the Mpro enzyme of SARS-CoV-2 (Farag et al., 2020).

\section{Results}

\section{Identification of genes differentially expressed upon infection with SARS-CoV-2}

Blanco-Melo et al. (2020) recently characterized the transcriptional response of the adenocarcinoma human alveolar basal epithelial cell line A549 after infection with SARS-CoV-2, which represents one of the pioneering cell models for COVID-19. We considered the genes upregulated $(N=100)$ and downregulated $(N=20)$ after infection according to DESeq2, under the false discovery rate of $5 \%$, as the transcriptional signature associated with COVID-19 (Supplementary Table 3). These gene sets were input into CMap to identify drugs that were empirically observed to reverse this transcriptional signature in the same cell line (A549), according to a connectivity mapping analysis using the L1000 repository.

\section{Connectivity mapping reveals drugs with repurposing potential for COVID-19}

We attempted to identify drugs with repurposing potential for COVID-19 based on the transcriptional signature associated with SARS-CoV-2 infection in cancer lung cells (Blanco-Melo et al., 2020). We observed 76 drugs with potential to reverse the transcriptional signature associated with SARS-CoV-2 infection $(\tau<-90.00)$. The top compound was the drug reserpine $(\tau=$ -99.94; full list, including L1000 compound identification numbers, in Supplementary Table 4). Other compounds negatively associated with the transcriptional signature of A549 cells after SARS-CoV-2 infection, included the quinine-derivative hydroquinidine $(\tau=-99.44)$, used for heart arrhythmia treatment; antibiotics like doxycycline $(\tau=-92.29)$ and butoconazole $(\tau=-92.07)$; and the antidepressants fluoxetine $(\tau=-99.20)$ and maprotiline $(\tau=-99.10)$. An important drug being considered for the treatment of COVID-19, chloroquine, had a weak negative connectivity score ( $\tau$ 
$=-7.84$ ), suggesting it does not have a strong ability to reverse the transcriptional signature associated with SARS-CoV-2 infection in A549 cells. A similar compound which received a lot of media attention, hydroxychloroquine, was not tested in this cell line as part of the L1000 repository, so it is not reported here.

\section{Molecular docking analyses}

Of the 76 drugs with $\tau<-90.00$, we observed that 26 were small compounds which were already FDA approved (Corsello et al., 2017). We ran simulations to predict their ability to bind to the catalytic sites of the SARS-CoV-2 main protease (Mpro) and RNA-dependent RNA polymerase (RdRp) enzymes, corroborating their repurposing potential, and providing preliminary evidence of an additional mode of action involving direct interactions with viral components. The PLANTS scores calculated here reflect the energy change when the drug comes together with the catalytic site of either the RdRp or Mpro enzymes, with more negative numbers suggesting a more likely drug-protein interaction (Table 1; details of analyzed compounds in Supplementary Table 1). Only one compound was associated with negative PLANTS scores $<-90.00$, flupentixol, suggesting it likely interacts with both SARS-CoV-2 enzymes tested (Figure 1). We also found other seven drugs with suggestive interactions with either of the viral enzymes tested (PLANTS scores < -80.00), including reserpine, fluoxetine, trifluoperazine, sunitinib, atorvastatin, raloxifene, and butoconazole. We did not find evidence to suggest that the remainder drugs from the connectivity mapping analysis, with $\tau<-90.00$, would bind directly to the viral enzymes tested (although they have the potential to reverse the transcriptional signature associated with infection).

\section{Cross-referencing with drugs suggested from other studies}

We cross-referenced the top hits from our connectivity mapping analysis $(\tau<-90)$ with those suggested for repurposing against COVID-19 by other studies (Supplementary Table 2). First, we analyzed potential overlaps with the 69 drugs suggested by Gordon et al. (2020) as potential treatment options for COVID-19, based on druggable human proteins that interact with all SARSCoV-2 proteins. The only overlapping FDA-approved drug we observed was metformin, associated with a highly negative connectivity score in our analysis $(\tau=-98.27)$. We did not identify a potential interaction between metformin and SARS-CoV-2 Mpro or RdRp enzymes, but Gordon et al. found that the human NDUF genes, which can be modulated by metformin, interact with the viral proteins Nsp7 and Orf9c. We found no drugs in our connectivity mapping analysis $(\tau<-90.00)$ that overlapped with the HIV drugs suggested for repurposing against COVID-19 by Chang et al. (2020), or with the top compounds predicted to bind to the Mpro of SARS-CoV-2 by Farag et al. (2020). 


\section{Discussion}

There have been several recent advances in the identification of epitopes for the design of a vaccine against SARS-CoV-2 ( $\mathrm{Li}$ et al., 2020), or drugs that could be useful for treating infected patients (Belhadi et al., 2020), but there is currently no licensed effective treatments against COVID-19. Using a data-driven approach, our study identified commonly prescribed FDA-approved drugs that are associated with transcriptional signatures opposite to that caused by infection with SARS-CoV-2 in A549 cells, including hydroquinidine, maprotiline, clomipramine, tamoxifen, and doxycycline. Additional candidates from the connectivity mapping analyses were further predicted to bind directly to SARS-CoV-2 key enzymes, or to host genes that interact with SARS-CoV-2 components (Gordon et al., 2020), corroborating their repurposing potential and providing preliminary evidence of other modes of action against COVID-19 involving interactions with the viral machinery. These drugs included flupentixol, reserpine, fluoxetine, trifluoperazine, sunitinib, atorvastatin, raloxifene, butoconazole, and metformin, which should be prioritized in basic and clinical studies, to test their ability to treat or prevent COVID-19.

An interesting compound we identified in the connectivity mapping analysis was hydroquinidine, a quinine-related drug used for treating arrhythmia (Hermida et al., 2004). Quinine-related compounds such as chloroquine and hydroxychloroquine received a lot of media attention recently, despite the variable evidence corroborating their efficacy against COVID-19 (Owens, 2020). We found that different quinine-related compounds were associated with varying degrees of likelihood to reverse the transcriptional signature associated with SARS-CoV-2 in A549 cells. More specifically, hydroquinidine $(\tau=-99.44)$, quinidine $(\tau=-74.22)$, quinine $(\tau=-37.83)$, and chloroquine ( $\tau=-7.84)$ showed a variable range of negative associations, while hydroquinine $(\tau=59.05)$ was associated with a positive $\mathrm{\tau}$ score.

Perhaps one of the most viable candidate drugs found in our study is doxycycline, a commonly prescribed antibiotic that works against a range of bacterial infections, and that has also been suggested to protect against malaria (Tan et al., 2011) and filarial parasites (Singh et al., 2016). A study also suggested that doxycycline improved lung function in patients with obstructive pulmonary disease, which was attributed to its inhibitory effect on matrix metalloproteinases and its anti-inflammatory properties (Dalvi et al., 2011). Alternatively, the antidepressants fluoxetine, maprotiline, and clomipramine, and the antipsychotics flupentixol, sulpiride, trifluoperazine, which have a rich pharmacology, were also suggested as drugs with repurposing potential against COVID-19. We observed that flupentixol, fluoxetine, and trifluoperazine were also predicted to bind directly to SARS-CoV-2 components. Interestingly, a study by Dyall et al. (2014) found that several neurotransmitter modulators, including clomipramine, had an effective antiviral activity against both Severe Acute Respiratory Syndrome (SARS) and Middle East Respiratory Syndrome (MERS) coronaviruses, SARS-CoV-1 and MERS-CoV, in infected Vero E6 cells. We also identified the 
insulin sensitizing drug metformin as an interesting candidate which is commonly prescribed for type 2 diabetes (Rojas and Gomes, 2013), and that has been previously suggested to reverse lung fibrosis in humans (Rangarajan et al., 2018). This is particularly relevant as a study of fifty-one COVID-19 patients showed that $19.6 \%$ of the individuals had pulmonary deformations due to fibrosis (Li and Xia, 2020). Our analyses also suggested that the estrogen modulators raloxifene and tamoxifen may be protective against COVID-19, which is interesting since it appears that females are less likely to develop COVID-19-related complications relative to males (Guan et al., 2020b). In addition, Dyall et al. (2014) also found that tamoxifen had an effective antiviral activity against SARS-CoV-1 and MERS-CoV in Vero E6 infected cells.

Our top compound, reserpine, has been discontinued in some countries, but a study found that this drug and six derivatives of it were active against SARS-CoV-1 in Vero E6 cells (Wu et al., 2004). Another study, which screened the L1000 database for compounds expected to reverse the effect of the knockdown of the coatomer protein complex beta 2 gene (COPB2) (Avchaciov et al., 2020), required for SARS-CoV-1 replication (de Wilde et al., 2015), also suggested reserpine as a potential treatment option for COVID-19. The hypothesis behind that study was that host COPB2 is also required for SARS-CoV-2 replication, since SARS-CoV-1 shares 79\% sequence identity with SARS-CoV-2 (Lu et al., 2020). Interestingly, our analysis provides some evidence that corroborates this hypothesis, since reserpine was the top compound we observed to reverse the transcriptional signature associated with SARS-CoV-2 infection in A549 cells. The fact that this drug is further predicted to bind to the main protease of SARS-CoV-2 makes it an interesting candidate for drug repositioning.

Our study is limited because we have no clinical evidence that the drugs identified here can successfully treat COVID-19 patients, or even prevent cellular pathology in response to SARS-CoV-2, as we did not test this directly. Therefore, it is possible that the drugs mentioned here may not be effective against COVID-19, and they should not be prescribed for this purpose prior to further clinical testing, nor be taken without a doctor's prescription. Further prospective or observational clinical research is now needed to determine if these drugs are effective for treating COVID-19, and if so, to determine which doses are required. Additional studies should also test whether the compounds identified here may have a protective effect against infection (e.g. through direct interaction with viral machinery, as captured by our molecular docking simulations), or act after infection (e.g. by reversing viral-induced gene expression signatures, as captured via connectivity mapping). Additionally, it is likely that the transcriptional signature associated with acute infection differs from that elicited by prolonged exposure to SARS-CoV-2, and therefore future analysis of converging transcriptional signatures from multiple time points may reveal other compounds of importance. Despite these limitations, we highlight novel FDA-approved drug 
candidates with repurposing potential against COVID-19, which we identified using a data-driven, hypothesis-free approach.

\section{Acknowledgements}

This work was partly enabled by a grant from the Coordenação de Aperfeiçoamento de Pessoal de Nível Superior (BEX1279/13-0) to R.R.R.D. We thank Dr. Daniel Bean, Dr. Robert E. Schwartz and Jeanne W. Vendruscolo for helpful discussions. The authors declare no conflicts of interest.

\section{References}

Avchaciov, K., Burmistrova, O., and Fedichev, P.O. (2020). Al for the repurposing of approved or investigational drugs against COVID-19. Research Gate, doi:doi: 10.13140/RG.2.2.20588.10886.

Baud, D., Qi, X., Nielsen-Saines, K., Musso, D., Pomar, L., and Favre, G. (2020). Real estimates of mortality following COVID-19 infection. The Lancet Infectious Diseases, doi:10.1016/S14733099(20)30195-X.

Belhadi, D., Peiffer-Smadja, N., Lescure, F.-X., Yazdanpanah, Y., Mentré, F., and Laouénan, C. (2020). A brief review of antiviral drugs evaluated in registered clinical trials for COVID-19. medRxiv, 2020.2003.2018.20038190, doi:10.1101/2020.03.18.20038190.

Blanco-Melo, D., Nilsson-Payant, B.E., Liu, W.-C., Møller, R., Panis, M., Sachs, D., Albrecht, R.A., and tenOever, B.R. (2020). SARS-CoV-2 launches a unique transcriptional signature from in vitro, ex vivo, and in vivo systems. bioRxiv, 2020.2003.2024.004655, doi:10.1101/2020.03.24.004655.

Chang, Y.-C., Tung, Y.-A., Lee, K.-H., Chen, T.-F., Hsiao, Y.-C., Chang, H., Hsieh, T.-T., Su, C.-H., Wang, S.-S., Yu, J.-Y., et al. (2020). Potential therapeutic agents for COVID-19 based on the analysis of protease and RNA polymerase docking. Preprints, doi:doi:

10.20944/preprints202002.0242.v1.

Corsello, S.M., Bittker, J.A., Liu, Z., Gould, J., McCarren, P., Hirschman, J.E., Johnston, S.E., Vrcic, A., Wong, B., Khan, M., et al. (2017). The Drug Repurposing Hub: a next-generation drug library and information resource. Nature medicine 23, 405-408, doi:10.1038/nm.4306.

COVID-19 Clinical Research Coalition (2020). Global coalition to accelerate COVID-19 clinical research in resource-limited settings. The Lancet, doi:doi: 10.1016/S0140-6736(20)30798-4.

Dalvi, P.S., Singh, A., Trivedi, H.R., Ghanchi, F.D., Parmar, D.M., and Mistry, S.D. (2011). Effect of doxycycline in patients of moderate to severe chronic obstructive pulmonary disease with stable symptoms. Ann Thorac Med 6, 221-226, doi:10.4103/1817-1737.84777.

de Wilde, A.H., Wannee, K.F., Scholte, F.E.M., Goeman, J.J., ten Dijke, P., Snijder, E.J., Kikkert, M., and van Hemert, M.J. (2015). A Kinome-Wide Small Interfering RNA Screen Identifies Proviral and Antiviral Host Factors in Severe Acute Respiratory Syndrome Coronavirus Replication, Including Double-Stranded RNA-Activated Protein Kinase and Early Secretory Pathway Proteins. Journal of Virology 89, 8318-8333, doi:10.1128/jvi.01029-15.

Dyall, J., Coleman, C.M., Hart, B.J., Venkataraman, T., Holbrook, M.R., Kindrachuk, J., Johnson, R.F., Olinger, G.G., Jr., Jahrling, P.B., Laidlaw, M., et al. (2014). Repurposing of clinically developed drugs for treatment of Middle East respiratory syndrome coronavirus infection. Antimicrobial agents and chemotherapy 58, 4885-4893, doi:10.1128/aac.03036-14. 
Farag, A., Wang, P., Ahmed, M., and Sadek, H. (2020). Identification of FDA Approved Drugs Targeting COVID-19 Virus by Structure-Based Drug Repositioning. ChemRxiv, doi:doi: 10.26434/chemrxiv.12003930.v2.

Gao, Y., Yan, L., Huang, Y., Liu, F., Zhao, Y., Cao, L., Wang, T., Sun, Q., Ming, Z., Zhang, L., et al. (2020). Structure of the RNA-dependent RNA polymerase from COVID-19 virus. Science (New York, NY), eabb7498, doi:10.1126/science.abb7498.

Gordon, D.E., Jang, G.M., Bouhaddou, M., Xu, J., Obernier, K., O’Meara, M.J., Guo, J.Z., Swaney, D.L., Tummino, T.A., Huettenhain, R., et al. (2020). A SARS-CoV-2-Human Protein-Protein Interaction Map Reveals Drug Targets and Potential Drug-Repurposing. bioRxiv, 2020.2003.2022.002386, doi:10.1101/2020.03.22.002386.

Guan, W.-j., Liang, W.-h., Zhao, Y., Liang, H.-r., Chen, Z.-S., Li, Y.-m., Liu, X.-q., Chen, R.-C., Tang, C.-I., Wang, T., et al. (2020a). Comorbidity and its impact on 1590 patients with Covid-19 in China: A Nationwide Analysis. European Respiratory Journal, 2000547, doi:10.1183/13993003.005472020.

Guan, W.-j., Ni, Z.-y., Hu, Y., Liang, W.-h., Ou, C.-q., He, J.-x., Liu, L., Shan, H., Lei, C.-I., Hui, D.S.C., et al. (2020b). Clinical Characteristics of Coronavirus Disease 2019 in China. New England Journal of Medicine, doi:10.1056/NEJMoa2002032.

Hermida, J.S., Denjoy, I., Clerc, J., Extramiana, F., Jarry, G., Milliez, P., Guicheney, P., Di Fusco, S., Rey, J.L., Cauchemez, B., et al. (2004). Hydroquinidine therapy in Brugada syndrome. Journal of the American College of Cardiology 43, 1853-1860, doi:10.1016/j.jacc.2003.12.046.

Ji, Y., Ma, Z., Peppelenbosch, M.P., and Pan, Q. (2020). Potential association between COVID-19 mortality and health-care resource availability. The Lancet Global Health 8, e480, doi:10.1016/S2214-109X(20)30068-1.

Keenan, A.B., Wojciechowicz, M.L., Wang, Z., Jagodnik, K.M., Jenkins, S.L., Lachmann, A., and Ma'ayan, A. (2019). Connectivity Mapping: Methods and Applications. Annual Review of Biomedical Data Science 2, 69-92, doi:10.1146/annurev-biodatasci-072018-021211.

Korb, O., Stutzle, T., and Exner, T.E. (2009). Empirical scoring functions for advanced proteinligand docking with PLANTS. J Chem Inf Model 49, 84-96, doi:10.1021/ci800298z.

Kunkel, S.D., Suneja, M., Ebert, S.M., Bongers, K.S., Fox, D.K., Malmberg, S.E., Alipour, F., Shields, R.K., and Adams, C.M. (2011). mRNA expression signatures of human skeletal muscle atrophy identify a natural compound that increases muscle mass. Cell Metab 13, 627-638, doi:10.1016/j.cmet.2011.03.020.

Lee, W.-Y., Lee, W.-T., Cheng, C.-H., Chen, K.-C., Chou, C.-M., Chung, C.-H., Sun, M.-S., Cheng, H.-W., Ho, M.-N., and Lin, C.-W. (2015). Repositioning antipsychotic chlorpromazine for treating colorectal cancer by inhibiting sirtuin 1. Oncotarget 6, 27580-27595, doi:10.18632/oncotarget.4768.

Li, L., Sun, T., He, Y., Li, W., Fan, Y., and Zhang, J. (2020). Epitope-based peptide vaccine design and target site characterization against novel coronavirus disease caused by SARS-CoV-2. bioRxiv, 2020.2002.2025.965434, doi:10.1101/2020.02.25.965434.

Li, Y., and Xia, L. (2020). Coronavirus Disease 2019 (COVID-19): Role of Chest CT in Diagnosis and Management. American Journal of Roentgenology, 1-7, doi:10.2214/AJR.20.22954.

Liu, J., Lee, J., Salazar Hernandez, M.A., Mazitschek, R., and Ozcan, U. (2015). Treatment of obesity with celastrol. Cell 161, 999-1011, doi:10.1016/j.cell.2015.05.011.

Lu, R., Zhao, X., Li, J., Niu, P., Yang, B., Wu, H., Wang, W., Song, H., Huang, B., Zhu, N., et al. (2020). Genomic characterisation and epidemiology of 2019 novel coronavirus: implications for virus origins and receptor binding. The Lancet 395, 565-574, doi:10.1016/S0140-6736(20)30251-8.

Onder, G., Rezza, G., and Brusaferro, S. (2020). Case-Fatality Rate and Characteristics of Patients Dying in Relation to COVID-19 in Italy. Jama, doi:10.1001/jama.2020.4683.

Owens, B. (2020). Excitement around hydroxychloroquine for treating COVID-19 causes challenges for rheumatology. The Lancet Rheumatology, doi:doi: 10.1016/S2665-9913(20)30089-8.

Rangarajan, S., Bone, N.B., Zmijewska, A.A., Jiang, S., Park, D.W., Bernard, K., Locy, M.L., Ravi, S., Deshane, J., Mannon, R.B., et al. (2018). Metformin reverses established lung fibrosis in a bleomycin model. Nature medicine 24, 1121-1127, doi:10.1038/s41591-018-0087-6. 
Rojas, L.B.A., and Gomes, M.B. (2013). Metformin: an old but still the best treatment for type 2 diabetes. Diabetology \& Metabolic Syndrome 5, 6, doi:10.1186/1758-5996-5-6.

Singh, Y., Srinivas, A., Gangwar, M., Meher, J.G., Misra-Bhattacharya, S., and Chourasia, M.K. (2016). Subcutaneously Administered Ultrafine PLGA Nanoparticles Containing Doxycycline Hydrochloride Target Lymphatic Filarial Parasites. Molecular Pharmaceutics 13, 2084-2094, doi:10.1021/acs.molpharmaceut.6b00206.

Subramanian, A., Narayan, R., Corsello, S.M., Peck, D.D., Natoli, T.E., Lu, X., Gould, J., Davis, J.F., Tubelli, A.A., Asiedu, J.K., et al. (2017). A Next Generation Connectivity Map: L1000 Platform and the First 1,000,000 Profiles. Cell 171, 1437-1452 e1417, doi:10.1016/j.cell.2017.10.049.

Subramanian, A., Tamayo, P., Mootha, V.K., Mukherjee, S., Ebert, B.L., Gillette, M.A., Paulovich, A., Pomeroy, S.L., Golub, T.R., Lander, E.S., et al. (2005). Gene set enrichment analysis: a knowledge-based approach for interpreting genome-wide expression profiles. Proc Natl Acad Sci U S A 102, 15545-15550, doi:10.1073/pnas.0506580102.

Tan, K.R., Magill, A.J., Parise, M.E., and Arguin, P.M. (2011). Doxycycline for Malaria Chemoprophylaxis and Treatment: Report from the CDC Expert Meeting on Malaria

Chemoprophylaxis. The American Journal of Tropical Medicine and Hygiene 84, 517-531, doi:doi: 10.4269/ajtmh.2011.10-0285.

Wu, C.-Y., Jan, J.-T., Ma, S.-H., Kuo, C.-J., Juan, H.-F., Cheng, Y.-S.E., Hsu, H.-H., Huang, H.-C., Wu, D., Brik, A., et al. (2004). Small molecules targeting severe acute respiratory syndrome human coronavirus. Proceedings of the National Academy of Sciences of the United States of America 101, 10012-10017, doi:10.1073/pnas.0403596101.

Wu, Z., and McGoogan, J.M. (2020). Characteristics of and Important Lessons From the Coronavirus Disease 2019 (COVID-19) Outbreak in China: Summary of a Report of 72314 Cases From the Chinese Center for Disease Control and Prevention. Jama, doi:10.1001/jama.2020.2648.

Zhang, L., Lin, D., Sun, X., Curth, U., Drosten, C., Sauerhering, L., Becker, S., Rox, K., and Hilgenfeld, R. (2020). Crystal structure of SARS-CoV-2 main protease provides a basis for design of improved $\alpha$-ketoamide inhibitors. Science (New York, NY), eabb3405,

doi:10.1126/science.abb3405. 

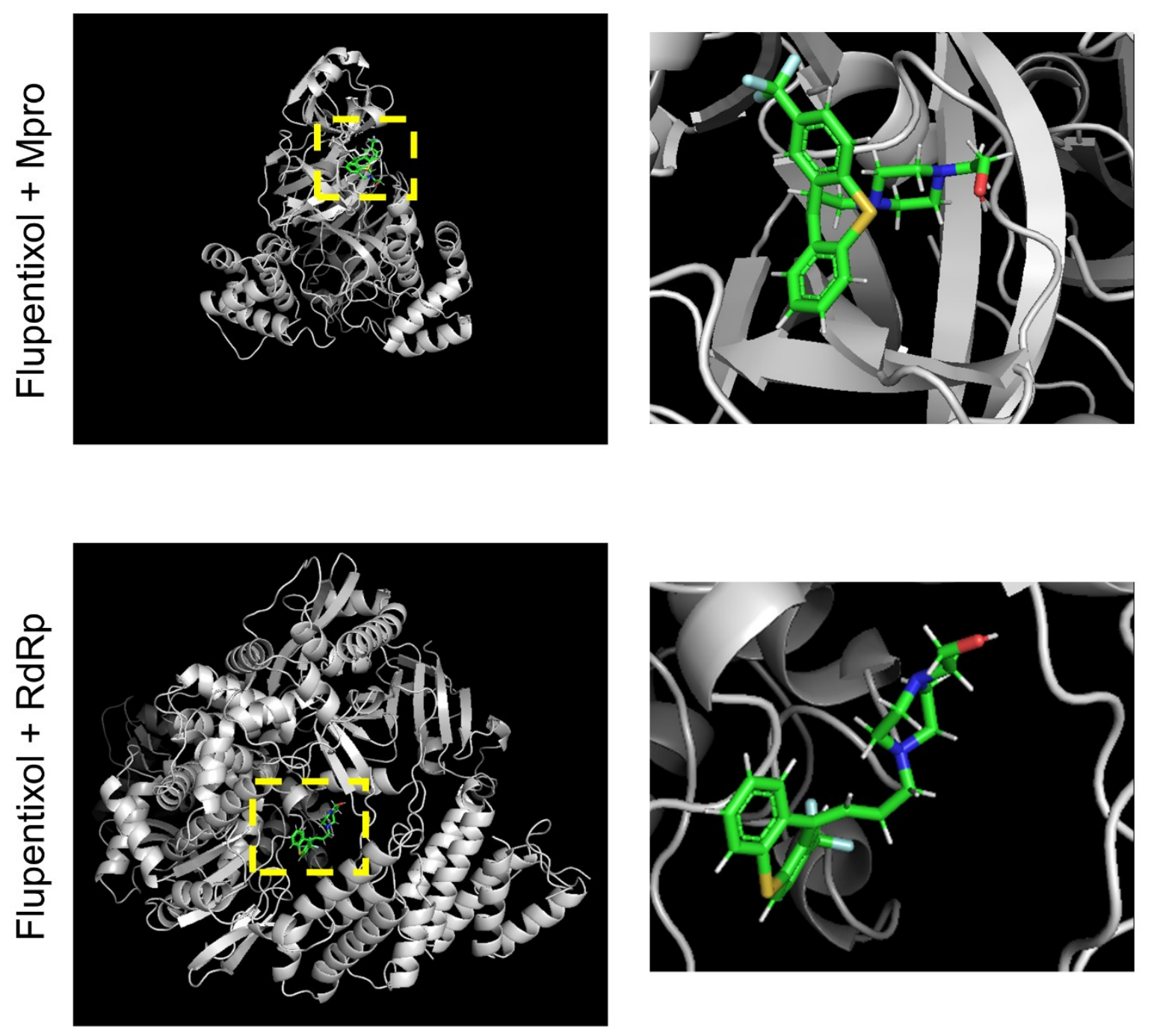

Figure 1. Representation of the predicted interactions between the antipsychotic flupentixol (in green) and the SARS-CoV-2 main protease (Mpro, upper panel) and RNA-dependent RNA polymerase (RdRp, lower panel) enzymes. Flupentixol was associated with a highly negative $\tau$ score $(\tau=-95.9)$ in the connectivity mapping analysis, suggesting it is associated with a transcriptional signature negatively correlated with SARS-CoV-2 infection. Furthermore, it was associated with low PLANTS scores in our chemoinformatic analyses, further suggesting it has the potential to bind directly to SARS-CoV-2 Mpro (-91.82) and RdRp (-91.70). 
Table 1. Molecular docking simulations of FDA-approved small compounds associated with protection against COVID-19, on the RNA-dependent RNA polymerase (RdRp) and Main protease (Mpro) enzymes of

\begin{tabular}{|c|c|c|c|c|}
\hline \multirow{2}{*}{$\begin{array}{l}\text { CMap } \\
\text { score }\end{array}$} & \multirow{2}{*}{ Drug Name } & \multirow{2}{*}{ Established Function } & \multicolumn{2}{|c|}{ PLANTS scores } \\
\hline & & & RdRp & Mpro \\
\hline-99.94 & reserpine & Vesicular monoamine transporter inhibitor & $\mathrm{N} / \mathrm{A}$ & -80.72 \\
\hline-99.44 & hydroquinidine & Antiarrhythmic & -68.79 & -79.05 \\
\hline-99.2 & fluoxetine & Selective serotonin reuptake inhibitor (SSRI) & -77.63 & -81.14 \\
\hline-99.1 & maprotiline & Norepinephrine reuptake inhibitor & -72.42 & -76.15 \\
\hline-98.87 & tamoxifen & Estrogen receptor antagonist & -57.40 & -63.05 \\
\hline-98.82 & dextromethorphan & Glutamate receptor antagonist & $\mathrm{N} / \mathrm{A}$ & -62.08 \\
\hline-98.77 & phensuximide & Succinimide antiepileptic & N/A & -66.02 \\
\hline-98.58 & sulpiride & Dopamine receptor antagonist & -72.36 & -64.34 \\
\hline-98.27 & metformin & Insulin sensitizer & -32.82 & -31.63 \\
\hline-97.9 & $\underline{\text { trifluoperazine }}$ & Dopamine receptor antagonist & -84.29 & -84.43 \\
\hline-96.82 & sunitinib & FLT3 inhibitor & -73.52 & -80.93 \\
\hline-96.78 & irsogladine & Phosphodiesterase inhibitor & -60.14 & -61.59 \\
\hline-96.65 & atorvastatin & HMGCR inhibitor & -89.22 & -82.17 \\
\hline-96.09 & raloxifene & Estrogen receptor antagonist & -84.32 & -87.57 \\
\hline-95.9 & flupentixol & Dopamine receptor antagonist & -91.70 & -91.82 \\
\hline-95.62 & alprenolol & Adrenergic receptor antagonist & -68.19 & -68.69 \\
\hline-94.89 & bosutinib & ABL inhibitor & -76.61 & -76.87 \\
\hline-94.76 & buflomedil & Adrenergic receptor antagonist & -63.00 & -67.89 \\
\hline-94.09 & guanfacine & Adrenergic receptor agonist & -59.95 & -61.08 \\
\hline-92.86 & epirizole & Cyclooxygenase inhibitor & -61.37 & -67.36 \\
\hline-92.75 & diloxanide & Protein synthesis inhibitor & -58.14 & -58.46 \\
\hline-92.7 & clomipramine & Serotonin transporter inhibitor (SERT) & -66.44 & -78.01 \\
\hline-92.7 & sulfacetamide & PABA antagonist & -55.60 & -54.92 \\
\hline-92.69 & norethindrone & Progesterone receptor agonist & N/A & -66.15 \\
\hline-92.29 & doxycycline & Bacterial 30 S ribosomal subunit inhibitor & -72.86 & -74.47 \\
\hline-92.07 & butoconazole & Bacterial cell wall synthesis inhibitor & -77.24 & -82.92 \\
\hline
\end{tabular}

N.B.: N/A indicates protein-ligand pairs that were extremely unlikely to occur according to PLANTS. PLANTS scores in blue represent ligand-protein interactions more likely to occur, with darker values indicating stronger evidence. 